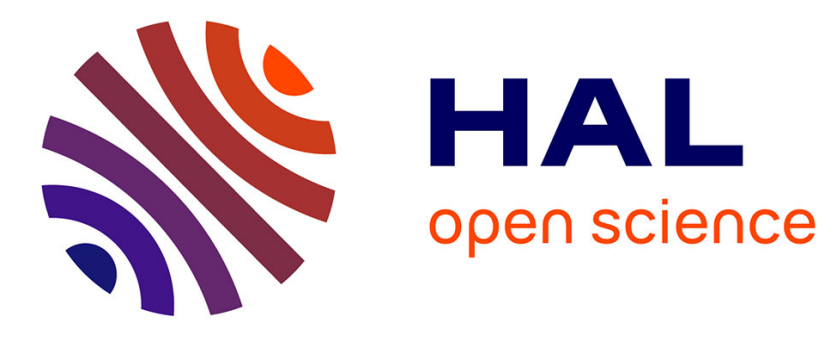

\title{
Downlink Packet Scheduling Algorithm Using Tabu Method in LTE Systems
}

Radhia Khdhir, Kais Mnif, Lotfi Kammoun

\section{To cite this version:}

Radhia Khdhir, Kais Mnif, Lotfi Kammoun. Downlink Packet Scheduling Algorithm Using Tabu Method in LTE Systems. 13th International Conference on Wired/Wireless Internet Communication (WWIC), May 2015, Malaga, Spain. pp.3-17, 10.1007/978-3-319-22572-2_1 . hal-01728814

\section{HAL Id: hal-01728814 https://hal.inria.fr/hal-01728814}

Submitted on 12 Mar 2018

HAL is a multi-disciplinary open access archive for the deposit and dissemination of scientific research documents, whether they are published or not. The documents may come from teaching and research institutions in France or abroad, or from public or private research centers.
L'archive ouverte pluridisciplinaire HAL, est destinée au dépôt et à la diffusion de documents scientifiques de niveau recherche, publiés ou non, émanant des établissements d'enseignement et de recherche français ou étrangers, des laboratoires publics ou privés.

\section{(c)(1)}

Distributed under a Creative Commons Attribution| 4.0 International License 


\title{
Downlink Packet Scheduling Algorithm using Tabu Method in LTE Systems
}

\author{
${ }^{1}$ Radhia Khdhir, ${ }^{2}$ Kais Mnif and ${ }^{1}$ Lotfi Kammoun \\ LETI Laboratory, \\ ${ }^{1}$ ENIS, University of Sfax, Tunisia \\ 2ENETCOM, University of Sfax, Tunisia \\ rkhdhir@gmail.com, kais.mnif@gmail.com,lotfikamoun2@gmail.com
}

\begin{abstract}
This paper addressed the problem of packet scheduling (PS) on the 3GPP Long Term Evolution (LTE) downlink (DL). The main contribution of this work was to propose a new scheduling and resource allocation scheme that deals with QoS requirements. The objectives of this proposed scheduler are to maximize the system's sum throughput, to allow a fair distribution of available RBs and to handle GBR and NGBR traffic in LTE downlink systems. The performance of the proposed approach was compared with previous resources allocation and scheduling algorithms such as Best-CQI, RR, and QoE downlink schedulers. Simulation results show that it is possible to achieve a considerable gain in both system's throughput and fairness.
\end{abstract}

Keywords: Scheduling; LTE; Downlink; Throughput; QoS

\section{1 introduction}

The Orthogonal Frequency Division Multiple Access (OFDMA) and Single Carrier Frequency Division Multiple Accesses (SC-FDMA) are techniques used for radio transmission and reception in LTE respectively for the Downlink and Uplink. The LTE system is expected to provide peak data rates in the order of $50 \mathrm{Mbit} / \mathrm{s}$ in Uplink (UL) with $20 \mathrm{MHz}$ spectrum allocation and $100 \mathrm{Mbps}$ in Downlink (DL), [1]. In LTE, the transmissions Downlink (DL) and uplink (UL) are organized through $10 \mathrm{~ms}$ time frames. Two frame types are supported: the first type is applicable for FrequencyDivision Duplexing (FDD) whereas the second is applicable for Time-Division Duplexing (TDD). The structure of frame type 1 is illustrated in figure 1. Each time frame $(10 \mathrm{~ms})$ is divided into 10 sub-frames equal to the duration of $1 \mathrm{~ms}$ called Transmission Time Interval (TTI). In FDD, Uplink and Dowlink transmissions are separated in the frequency area [2]. The structure of frame type 2 is illustrated in figure 2. Each frame $(10 \mathrm{~ms})$ is divided into two sub-frames of $5 \mathrm{~ms}$. Each sub-frame contains a special sub-frame and 4 other sub-frames. The duration of the special subframe is $1 \mathrm{~ms}$ and contains three fields of DwPTS (Downlink Pilot Time Slot), GP (Gurand Period) and UpPTS (Uplink Pilot Time Slot).

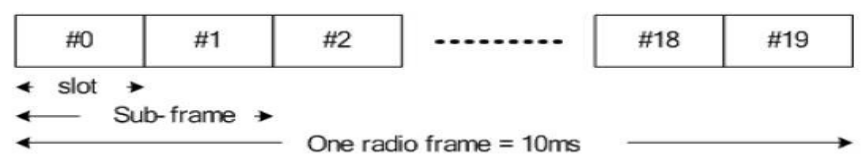

Fig.1. Frame structure type 1[2] 
In the frame type 2, there are 7 different patterns of uplink-downlink switching, termed uplink-downlink configurations 0 through 6. In LTE Downlink (DL), the total bandwidth is divided into multiple sub-bandwidths. These are regrouped in PRBs (Physical Resources Block). A PRB is defined by a couple frequency and time domains. In fact, a RRB is $0.5 \mathrm{~ms}$ in length (one slot in the time domain) and contains a contiguous set of 12 subcarriers $(180 \mathrm{kHz}$ in the frequency domain) from each OFDM symbol, as shown in figure 3. Therefore, this PRB is the basic transmission unit of a user's data in both uplink and downlink. The number of PRB's in a frequency domain is between 6 and 110 [3]. The LTE standard defines nine Qualities of service class identifiers (QCI), four GBR (Guaranteed Bit Rate) and five non-GBR [4], while providing a Quality of Service (QoS) for multiple types of traffic.

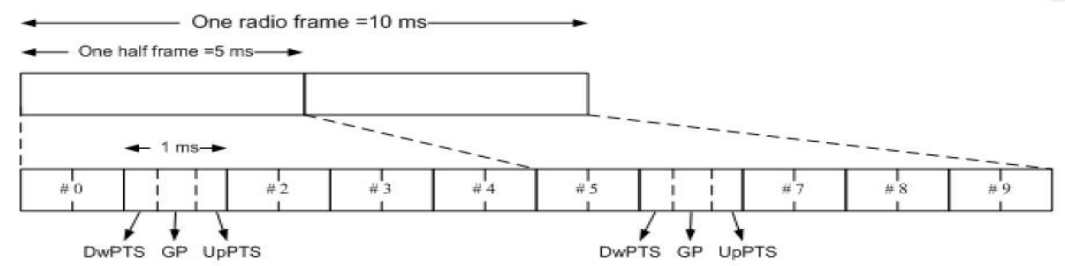

Fig.2. Frame structure type 2[2]

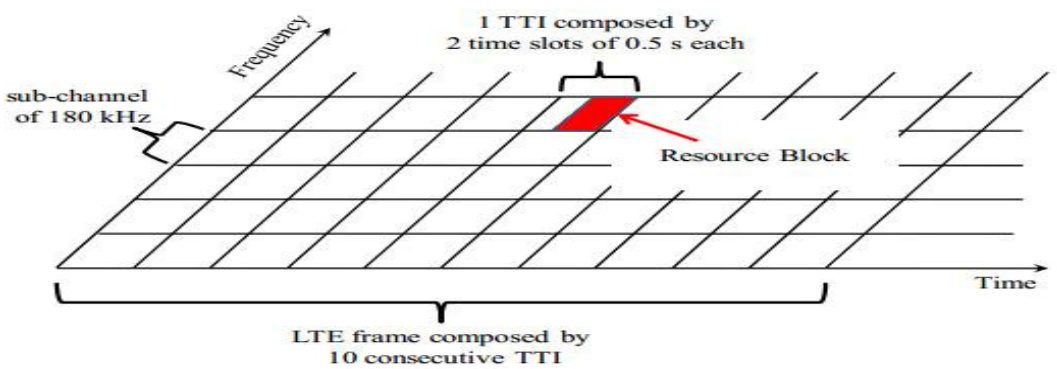

Fig.3. Downlink resource grid [13]

In the LTE standard, there are no defined specific scheduling algorithm nor allocation algorithm. Consequently, an LTE scheduling and allocation algorithm has been discussed by many researchers from both academic and industrial fields. An LTE downlink scheduler selects a set of UEs to be scheduled in the following TTI based on their QoS requirements and many other conditions. In this work, the main contribution was to propose a new scheduling scheme for OFDMA system, by considering GBR and non-GBR traffic, the maximization throughput and the user fairness into consideration.

This paper is organized as follows: section 2 presented the review of existing downlink scheduling algorithms in OFDMA. The system and our scheduling algorithm were introduced in Section 3. The simulation results and discussions were detailed in section 4. We finally drew the conclusion of our work in Section 5. 


\section{RELATED WORK}

LTE downlink scheduling algorithms have been discussed by many authors. From the literature overview, in the Best Quality Indication (BCQI) scheduling the user who has the best channel quality gets served. But, the UEs that suffer from bad channel conditions will never be served by this scheduler [5]. So, UEs that are far away from eNodeB never get the resources. Therefore, the BCQI achieve better throughput results but it is poor in terms of fairness. Authors in [6], relied on an opportunistic approach to allocate the radio resources. Mushtaq's focused on the Mean Opinion Score MOS, average throughput, and channel condition for GBR and non-GBR traffic to calculate the UEs priorities. To evaluate the priorities of the UEs, authors relied on the Quality of Experience (QoE) metrics. The authors proposed in [7] two scheduling algorithms based in MIMO-FDPS (Multiple Input Multiple Output - Frequency Domain Packet Scheduling) problem. The main motivation of their work cannot provide QoS satisfaction for different users. In [8], the authors proposed a new scheduling algorithm namely the Quality-aware DRX (Q-DRX) scheme. The objective of this proposed algorithm is not only to improve the QoS but also to save the UE's power. Q-DRX is evaluated in terms of Throughput Fairness Index, System Throughput, Packet Loss Rate and Packet Delay. The performance of the Q-DRX scheme is better than other scheduling algorithms such as RR, BCQI and PF. Lin and Yue in [9], proposed a novel algorithm for the OFDMA system namely ChannelAdapted and Buffer-Aware (CABA). The CABA algorithm, which is based on the QoS for Real Time and Non-Real Time services to schedule the UE's. In contrast, using the $\mathrm{CABA}$ algorithm, the packet loss rate can increase because this scheduler does not consider the packet delays. In the work referenced by [10], a new packet scheduling scheme was presented. In this scheme, the authors combined both of the time domain and frequency domain scheduling. The aim of this algorithm is to maximize the throughput guaranteeing the minimum delay packet for each UE. The limit of this proposed scheme does not base on the channel condition allowing the RB's to UE's. In [11], a scheduling method is proposed. This method based on optimal DRX parameters guaranteeing the delay and power saving constraints, but does not rely on any other parameters like QoS, fairness, throughput etc. Also this proposed method is applied with two users only. The following table gives a summary of the mentioned algorithms.

\begin{tabular}{|c|l|l|}
\hline Ref & Strengths & Limitations \\
\hline$[5]$ & $\begin{array}{l}\text { The Best Quality Indication (BCQI) scheduling is proposed. } \\
\text { This scheduler served the UE's which have the best channel } \\
\text { quality. }\end{array}$ & $\begin{array}{l}\text { Does not consider different } \\
\text { levels of fairness: the UE's } \\
\text { that suffer from bad channel } \\
\text { conditions will never be } \\
\text { served by this scheduler. }\end{array}$ \\
\hline$[6]$ & $\begin{array}{l}\text { Authors proposed a new downlink scheduling algorithm. } \\
\text { This algorithm considers many conditions Mean Opinion } \\
\text { Score MOS, average throughput, channel condition, and } \\
\text { Guaranteed Bit Rate (GBR) non-GBR traffic to calculate } \\
\text { the priorities of UEs. }\end{array}$ & \\
\hline
\end{tabular}




\begin{tabular}{|c|l|l|}
\hline [7] & $\begin{array}{l}\text { Two scheduling algorithms based in MIMO-FDPS } \\
\text { (Multiple Input Multiple Output-Frequency Domain Packet } \\
\text { Scheduling) problem are proposed. }\end{array}$ & $\begin{array}{l}\text { Does not consider the service } \\
\text { differentiation }\end{array}$ \\
\hline$[8]$ & $\begin{array}{l}\text { The authors proposed a new scheduling algorithm namely } \\
\text { the Q-DRX scheme. The objective of this proposed } \\
\text { algorithm is to improve QoS and power. Q-DRX is evaluated } \\
\text { in terms of Throughput Fairness Index, System Throughput, } \\
\text { Packet Loss Rate and Packet Delay. }\end{array}$ & None \\
\hline$[9]$ & $\begin{array}{l}\text { Proposed a novel algorithm for the OFDMA system CABA. } \\
\text { The CABA based on the QoS for Real Time and Non-Real } \\
\text { Time services to schedule the UE's. }\end{array}$ & $\begin{array}{l}\text { Does not consider the packet } \\
\text { delays. So the packet loss rate } \\
\text { can increase. }\end{array}$ \\
\hline$[10]$ & $\begin{array}{l}\text { Presented a new packet scheduling scheme. The aim of this } \\
\text { algorithm is to maximize the throughput guaranteeing the } \\
\text { minimum delay packet for each UE. }\end{array}$ & $\begin{array}{l}\text { Does not rely on the channel } \\
\text { condition to assign the RB's to } \\
\text { UE's. }\end{array}$ \\
\hline$[11]$ & $\begin{array}{l}\text { A scheduling method is proposed. This method based on } \\
\text { optimal DRX parameters guaranteeing the delay and power } \\
\text { saving constraints. }\end{array}$ & $\begin{array}{l}\text { Does not rely on any other } \\
\text { parameters like QoS, fairness, } \\
\text { throughput. This method is } \\
\text { simulated with only two UE's }\end{array}$ \\
\hline
\end{tabular}

To sum up, The objectives of downlink schedulers are to be able to share the total system bandwidth fairly according to the QoS requested by each user, to minimize the power consumption while ensuring feasible algorithm complexity and system scalability and maximizing the throughput with respect to the QoS.

\section{System Model and Proposed Scheduling Algorithm}

\subsection{System Model}

In LTE, the evolved NodeB (eNodeB) is the entity in charge of performing the resource allocation and the Packet Scheduling task. The PS is considered the most important step of RRM (Radio Resource Management) feasible per eNodeB [12]. LTE PS comprises timedomain (TD) and frequency domain (FD) scheduling algorithms. The TD scheduler selects a set of UEs requests to be served in the next TTI based on their QoS requirements. The selected set of UEs requests is passed to the FD scheduler that determines the RBs that should be allowed to them relying on the channel quality. The scheduling algorithm of our system is shown in figure 4

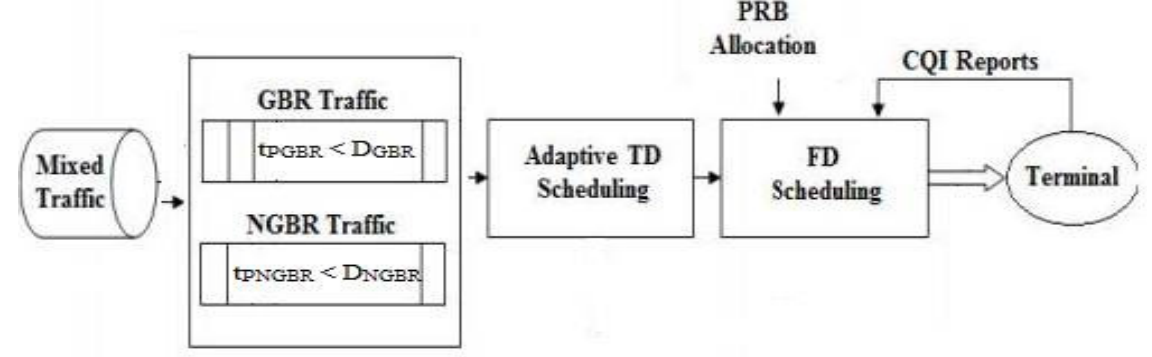

Fig.4. DPSA-Tabu system model 
As shown in figure 4, the packets coming in mixed traffic will be classified into two classes: a GBR class and NGBR class. So, each type of packet will be delivered in an independent queues: GBR queue and NGBR queue. These two queues will be served on the basis of opt-tabu algorithm [14] (see section 3.2.1). We define $\boldsymbol{t}_{\boldsymbol{p} G B R}$ the GBR packet delay and $\mathbf{D}_{\text {GBR }}$ is the delay budget which is the upper delay bound of the GBR traffic. Therefore, each packet delay of a GBR type must not exceed this delay budget. Same reasoning for NGBR class: $\boldsymbol{t}_{\boldsymbol{p} \text { NGBR }}$ is the NGBR packet delay and $\boldsymbol{D}_{\boldsymbol{N G B R}}$ is the delay budget which is the upper delay bound of the NGBR traffic.

Let us consider a cellular network where downlink OFDMA system bandwidth is divided into $m$ RBs, one eNodeB and $n$ active UEs. In this research, we consider an infinitely backlogged model in which there is always data available for the service for each UE. The eNodeB can allocate $m$ RBs to a set of $n$ UEs. Each time slot, multiple RBs can be assigned to a single UE. In other words, each PRB however can be assigned to one user at most.

\subsection{Proposed Scheduling algorithm}

In this subsection, we first briefly explained the concept of opt-tabu algorithm referenced in [14]. Then, we presented the downlink packet scheduling algorithm using the Tabu method based on the opt-tabu criterion. This novel scheduling algorithm for downlink LTE system, namely DPSA-Tabu (Downlink Packet Scheduling Algorithm) used the Tabu method. The performance of this proposed algorithm was presented in section 4 .

\subsubsection{Opt-Tabu scheduler [14]}

The scheduler receives the matrix $M$ as input, with $[n \times m]$ as dimensions, where $n$ is the number of users (UEs) and $m$ is the number of PRBs. The matrix values are calculated according to the "Proportional fair scheduling" metric (PF) [15]. For each user, we calculate the amount of $M_{i, c}$. where:

- $\quad M_{i, c}=\frac{r_{U E_{i}}^{P R B_{c}}}{R_{U E_{i}}(t)}$

- $\quad r_{U E_{i}}^{P R B_{c}}$ the throughput of instantaneous channel for user $\mathrm{UE}_{\mathrm{i}}(i=1,2, \ldots, n)$ if we assign it a $P R B_{c}(i=1,2, \ldots, m)$ at time $t$.

- $R_{U E_{i}}(t)$ is the average instantaneous throughput

- $R_{U E_{i}}(t)=\frac{1}{N_{T}} R_{U E_{i}}(t-1)+\frac{1}{N_{T}} r_{U E_{i}}^{P R B_{C}}(t-1)$

- $\quad N_{T}$ as a response time of low-pass filter

The algorithm follows the Tabu method (for more details about this method see [16] and [17]) to search a better allocation through ensuring the maximum global throughput. So the optimization problem is to optimize the allocation $s$. Let's define $f(s)$ as the function that calculates the global throughput at each iteration or each assignment between $\mathrm{UE}_{\mathrm{i}}$ and $\mathrm{PRB}_{\mathrm{c}}$. so:

Let's define:

$$
f(s)=\sum_{i=1}^{n} \sum_{c=1}^{m} M_{i, c}, \text { for }(i=c)
$$


- $\quad s$ is the current solution (current allocation),

- $s^{*}$ is the best-known solution (best allocation),

- $\quad N(s)$ is the possible permutations (between two UEs),

- $\quad T$ is the Tabu list (tabu memory),

- $f(s)$ is the function which calculates the throughput system.

The steps of the algorithm are as follows:

Step 1: Assign PRBs to UEs at random: $U E_{1} \leftarrow P R B_{1} ; U E_{2} \leftarrow P R B_{2} \ldots U E_{n} \leftarrow P R B_{m}$ and calculate the matrix values $M_{i, c}=\frac{r_{U E_{i}}^{P R B_{C}}}{R_{U E_{i}}(t)}$ for each $P R B_{c}$.

Step 2: Save the allocation (in step1) and calculate the global system throughput $f(s)$ (in this paper, we define the global system (uplink) throughput as the sum of all UE's instantaneous throughput in the uplink divided by the average instantaneous throughput). So we compute the sum of the diagonal matrix $M$ to calculate the global system throughput. We chose the diagonal of the matrix $M$ because one resource block can be assigned to one UE in each slot

Step 3: Follow the Tabu method in order to improve the allocation carried out in Step1: swap the rows of matrix $M$ (for example, Assign $\mathrm{PRB}_{1}$ to user $\mathrm{UE}_{2}$ and $\mathrm{PRB} 2$ to user UE1opposite to the initial case (step1)). Then, calculate $f(s)$ for this allocation

Step 4: After the comparison with the preceding allocation (allocation1 in step1) and current allocation (allocation 2 in step3), if allocation 2 is better than allocation1 (i.e. $\left(f(s)>f^{*}\right)$ then we consider the optimal allocation $\left(s^{*}\right)$ our current solution and save the permutation in the subset $\mathrm{N}(\mathrm{s})$. Else we consider the optimal solution $\left(s^{*}\right)$ the preceding allocation and save the permutation in the $\mathrm{T}$ Tabu memory. This Tabu memory contains the achieved permutations which have not improved the previous allocations. So it is useless to revisit this configuration.

Step 5: Repeat step3 and step4 until the end of the number of users $\mathrm{n}$ by searching the best global system throughput $f^{*}$

Step 6: Find the best allocation $\left(s^{*}\right)$ which corresponds to $f^{*}$ (the maximum calculated throughput).

Step 7: Apply $s^{*}$ allocation (the optimal allocation) which corresponds to $f^{*}$ )

Step 8: Repeat Step 2 up to Step 7 until all PRBs are allocated.

3.2.2 The Downlink Packet Scheduling Algorithm using the Tabu method (DPSA-Tabu)

The objective of our work is to propose and model a novel downlink scheduling algorithm not only by keeping a high system throughput but also guaranteeing the QoS constraints for all UE's.

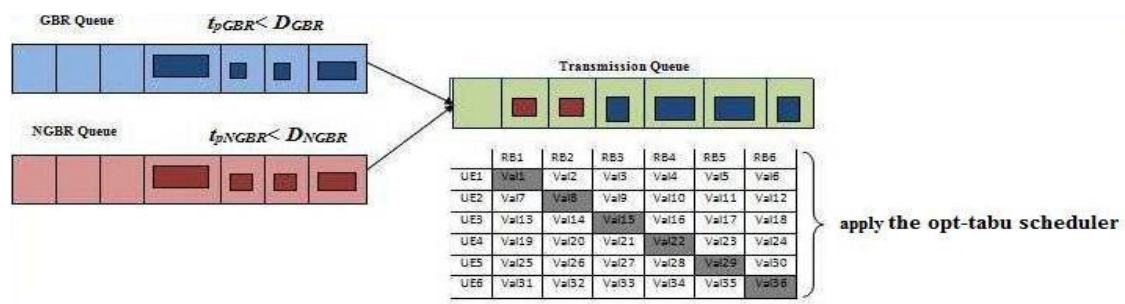

Fig.5. An illustration of downlink transmissions considering 6 RBs and 6 UEs including GBR packets and NGBR packets 
As mentioned in section 3.1, the packets coming in mixed traffic are then classified into two classes: a GBR and NGBR class. These two queues will be served on the basis of opt-tabu [14]. We define $\boldsymbol{t}_{\boldsymbol{p} \boldsymbol{G B \boldsymbol { R }}}$ the delay of packet $\boldsymbol{p}$ of GBR traffic and $\boldsymbol{D}_{\boldsymbol{G B R}}$ is the delay budget which is the upper delay bound of GBR traffic. Therefore, each packet delay of a GBR type must not exceed this delay budget $\boldsymbol{t}_{\boldsymbol{p} G \boldsymbol{B} \boldsymbol{R}}<\boldsymbol{D}_{\boldsymbol{G B R}}$. Therefore, if this condition is not respected the packet will be removed from the GBR queue.

The same reasoning is applied for the NGBR class: $\boldsymbol{t}_{\boldsymbol{p N G B R}}$ is the delay of packet $\boldsymbol{p}$ of NGBR traffic and $\boldsymbol{D}_{N G B R}$ is the delay budget which is the upper delay bound of the NGBR traffic $\boldsymbol{t}_{\boldsymbol{p} N G B R}<\boldsymbol{D}_{N G B R}$. (i.e: $\boldsymbol{D}_{G B R}<\boldsymbol{D}_{N G B R}$ ). Here, we define $\boldsymbol{l e n g}_{G B R}$ the length of GBR queue and $\boldsymbol{\rho}_{\text {GBR }}$ the threshold size of the GBR queue. For the GBR packets buffered in a GBR queue, they are delivered into every slot. For the NGBR packets buffered in the NGBR queue, they are delivered whenever the condition $\left(\boldsymbol{l e n g}_{G B R}<\boldsymbol{\rho}_{\mathrm{GBR}}\right)$. Then schedule the GBR and the NGBR packets admitted to be delivered based on the opt-tabu criterion.

The steps of the algorithm are as follows:

Step 1: Deliver GBR packets buffered in GBR queue if $\boldsymbol{t}_{\boldsymbol{p} \boldsymbol{G B R}}<\boldsymbol{D}_{\boldsymbol{G B R}}$ in the current scheduling $\mathrm{S}$

Step 2: Deliver NGBR packets buffered in NGBR queue if $\boldsymbol{t}_{\boldsymbol{p N G B R}}<\boldsymbol{D}_{N G B R}$ and $\boldsymbol{l e n g}_{G B R}<$ $\boldsymbol{\rho}_{\mathrm{GBR}}$ in iteration $\mathrm{s}+1$.

Step 3: Apply the opt-tabu algorithm with UEs which have the admitted packets to deliver (in step1 and 2).

Step4: Drop the GBR traffic if their delay constraint is violated with respect $\boldsymbol{D}_{\boldsymbol{G B R}}$. Drop the NGBR traffic if their delay constraint is violated with respect $\boldsymbol{D}_{N G B R}$.

Step5: Repeat step1 up to step4 until all PRBs are allocated or the two GBR and NGBR queues are empty.

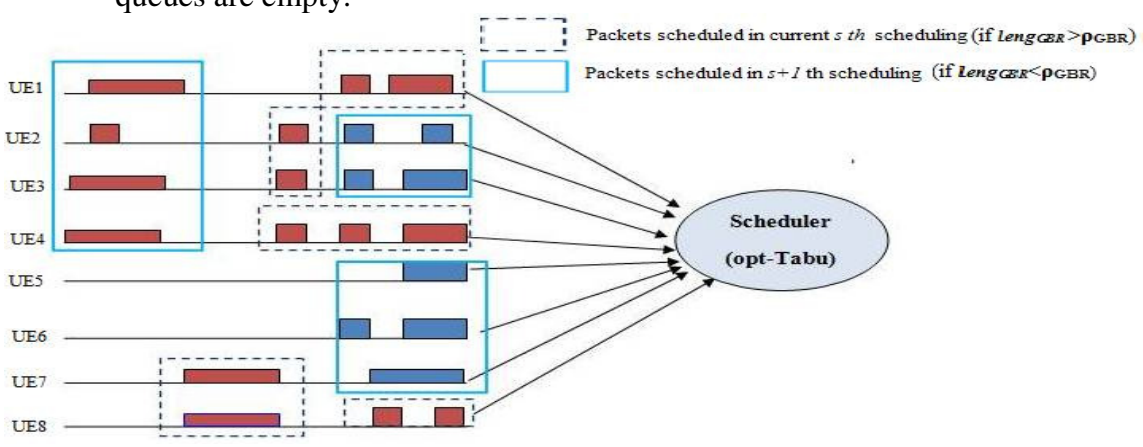

Fig.6. An illustration of downlink transmissions considering 8 UEs including GBR packets (UE1, UE2, UE3, UE4, UE7 and UE8) and NGBR packets (UE2, UE3, UE5, UE6 and UE7)

Figure 6 shows an example of a transmission scenario for our proposed algorithm considering the packets of type GBR and NGBR traffic and 8 UEs.

Here we suppose two cases: 
Case 1: in the current $s$ scheduling, Assume that leng $_{G B R}>\boldsymbol{\rho}_{\mathbf{G B R}}$. Thus, the users have GBR packets (UE1, UE2, UE3, UE4, UE7 and UE8) can only deliver their packets in the current iteration $\mathrm{s}$ by our definition.

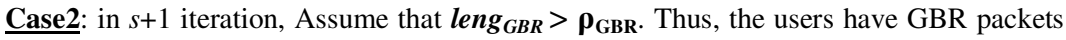
(UE1, UE2, UE3, UE4, UE7 and UE8) and NGBR packets (UE2, UE3, UE5, UE6 and UE7) can deliver their packets in $s+1$ scheduling by our definition.

The prediction of the fluctuation of the traffic is plotted in figure 7. So, we can firstly calculate the steady state probability of the GBR and NGBR queue length and we can determine the leng $_{G B R}$ (length of GBR traffic queue). Thus, make the scheduling decision: case1 and case2.

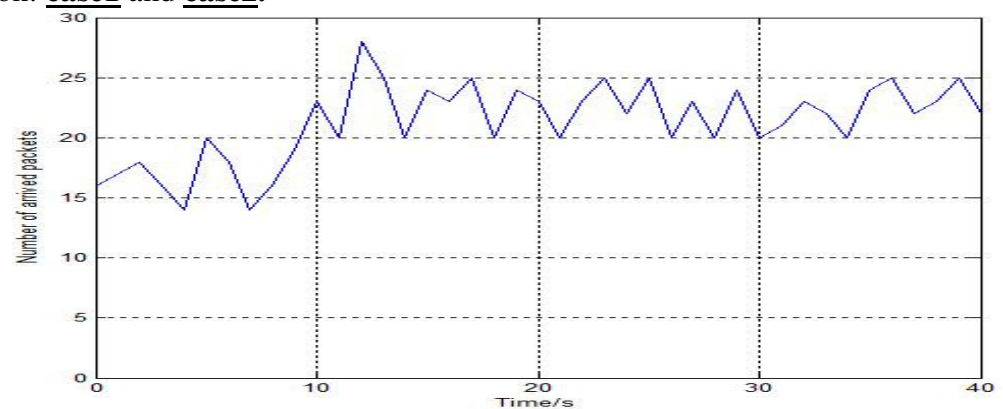

Fig.7. Number of arrived packets

\section{Simulation results and discussions}

In this section we presented the simulation results obtained by applying the algorithms explained in section 3 .

\subsection{Simulation Parameters}

In order to view the performance of the proposed scheduler (DPSA-Tabu) we use traces generated in 3GPP deployment evaluation [18]. The configuration parameters used in this simulation are shown in Table1.

Table1. Simulation parameters

\begin{tabular}{l||l}
\hline \multicolumn{1}{l||}{ Parameter } & Setting \\
\hline System bandwidth & $10 \mathrm{MHz}$ \\
Type of system & Single cell \\
Subcarrier spacing & $15 \mathrm{KHz}$ \\
Number of subcarriers per PRBs & 12 \\
Number of available PRB, & 50 \\
Transmission time interval (TTI) & $1 \mathrm{~ms}$ \\
Total number of used subcarriers & 600 \\
Carrier frequency & $2.5 \mathrm{GHz}$ \\
Frame duration & $10 \mathrm{~ms}$ \\
Link adaptation ACM Modulation & BPSK, QPSK,16-QAM, 64-QAM \\
Scheduling algorithms & RR, Best CQI, QoE scheme, DPSA-Tabu \\
&
\end{tabular}




\subsection{Simulation Results}

In this subsection, we presented the results obtained from the simulation that were achieved on the scheduler DPSA-Tabu. The conducted simulation was designed to analyze the following aspects of the system performance under different scenarios.

\subsubsection{System Throughput}

The system throughput is measured as the amount of user data carried by the system (total number of bits transmitted from the UEs) over the total simulation time. It is generally expressed in bits per second. The system throughput is calculated as follows:

$$
\boldsymbol{t h}_{\text {sys }}=\frac{\sum_{i=0}^{n b_{1} p c k} P \text { Packet_size } e_{i}}{T_{\text {sim }}}
$$

Where Packet_size $e_{i}$ the size of packet $i$ in Mbit, nb_pck is the number of packets transmitted during the simulation and $T_{\text {sim }}$ is the simulation time measured in seconds.

The average system throughput of RR, Best CQI, QoE scheme and DPSA-Tabu algorithms versus a number of UEs is shown in figure 8 . We can conclude that DPSA-Tabu provides the highest throughput. This is explained by our proposed scheme (DPSA-Tabu) that allocates the RBs to UEs with the best channel conditions and because it relies on the opt-tabu criterion to schedule the packets: the opt-Tabu considers the first objective is to maximize the total system throughput [14]. We can classify DPSA-Tabu scheme among the QoS-aware schemes. This category of algorithms distinguishes between different UEs and assigns less RBs to low priority applications (in this case less resource for the packets of type NGBR Traffic). In contrast, the QoS-aware algorithms give more resource for the best priority applications (in this case the packets of type GBR traffic) [19].

The result shows that the system throughput of the Best CQI algorithm is better compared to the QoE and RR, because this algorithm selects only the UEs which have the best channel conditions.

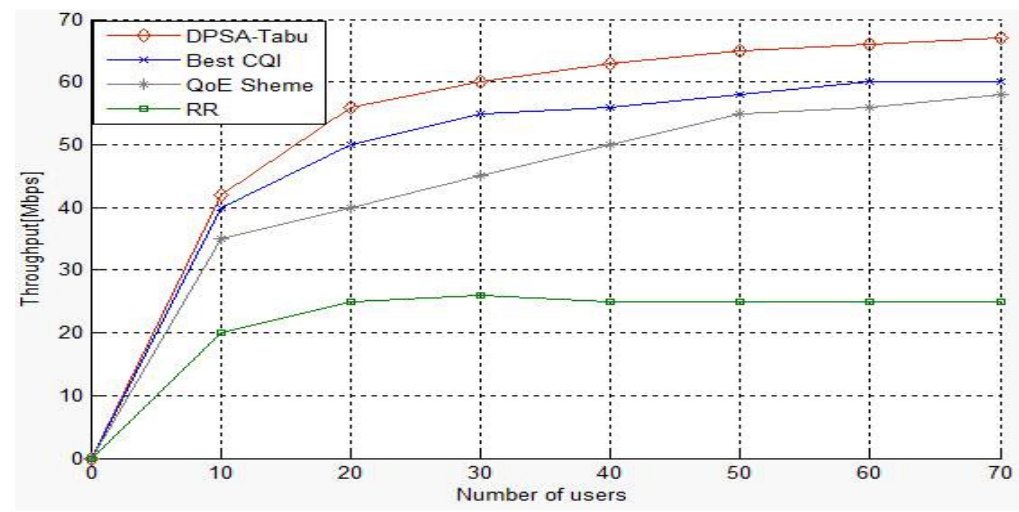

Fig.8. System throughput 


\subsubsection{Packet Loss Rate (PLR)}

The Packet Loss Rate is the ratio of the number of packets lost to the number of packets sent:

\section{Number of packets lost Number of packets sent}

The Packet Loss Rate is shown in figure 9. The performance of RR scheduler in terms of packet loss rate is a better than the other downlink scheduling algorithms. This is normal because the RR assigns RBs cyclically. Thus, each UE has the opportunity to get radio resources.

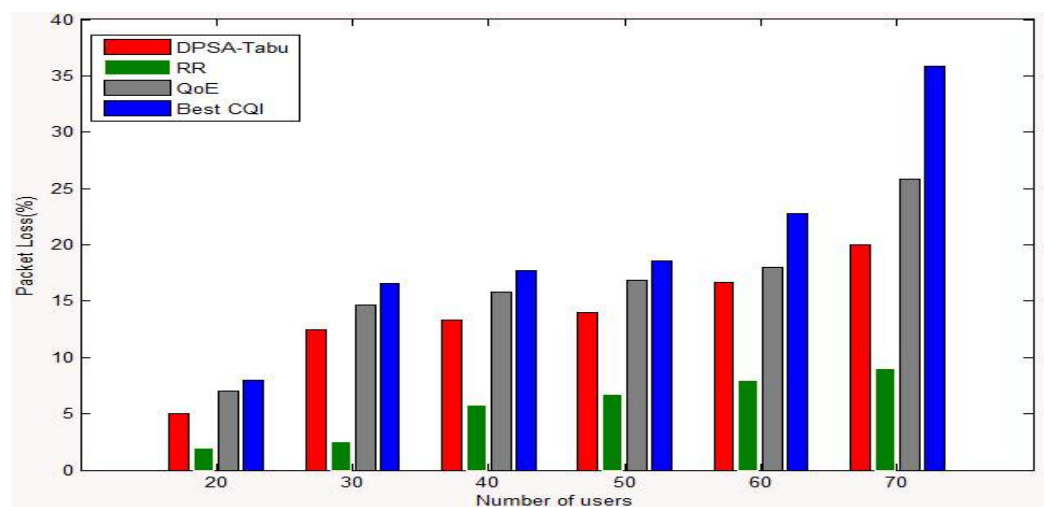

Fig.9. Packet Loss Rate

The DPSA-Tabu is in the second position. This is explained by the fact that if a packet delay exceeds the delay threshold $\left(\boldsymbol{D}_{\boldsymbol{G B R}}\right.$ and $\left.\boldsymbol{D}_{N G B R}\right)$ then it is classified to be the lost packet. Our proposed scheme is better than QoE and Best CQI schemes: the Best CQI BCQI has lowest performance. Because it allocates the RBs to UEs with the best channel conditions, while the packets of other UEs get lost due to insufficient resources.

\subsubsection{Fairness Index}

The fairness of the approaches is evaluated by the Jain's fairness index [20]. In our context, fairness index can be calculated as:

$$
F\left(\operatorname{res}_{1}, \operatorname{res}_{2}, \ldots \operatorname{res}_{n}\right)=\frac{\left(\sum_{j=1}^{n} \operatorname{res}_{j}\right)^{2}}{n \times \sum_{j=1}^{n}\left(\operatorname{res}_{j}\right)^{2}}
$$

Where $n$ the total number of UEs and res $_{\mathrm{j}}$ is the number of resources assign to UE $j$. Jain's fairness index returns a value between 0 and 1, 1 represents the highest fairness in the system.

Figure 10 shows the results of fairness for the schedulers RR, Best CQI, QoE and DPSA-Tabu. The maximum Jain's fairness index is obtained by an RR scheduler. 
This is normal because the RR assigns RBs cyclically so that the same throughput is guaranteed for all UEs. The simulation results show that DPSA-Tabu attained interesting results compared to RR scheme. The QoE Scheme is in third position: it tries to increase the user perception (MOS) while considering the throughput requirement of $\mathrm{RT}$ users.

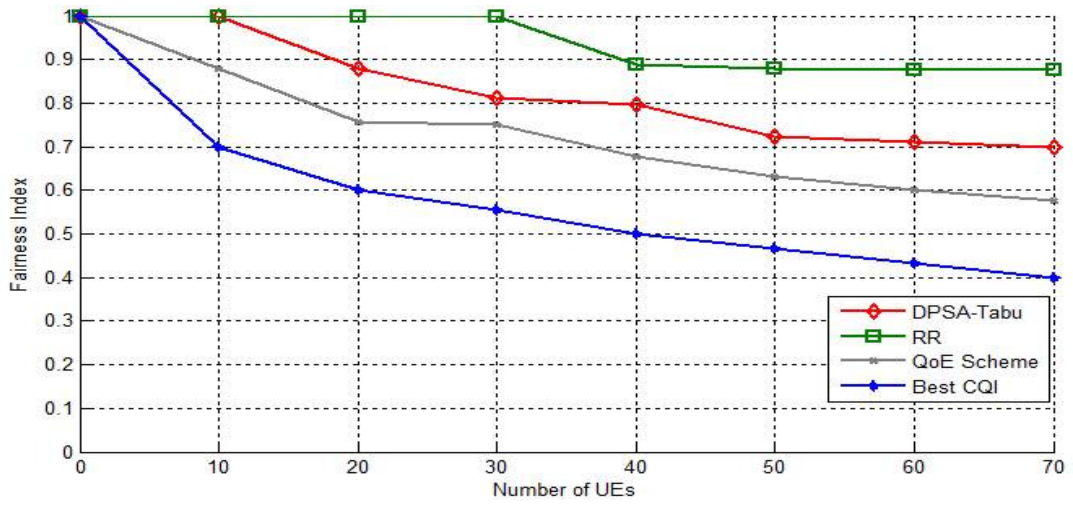

Fig.10. Fairness index

The Best CQI has a low fairness index compared to RR, QoE and DPSA-Tabu because the UEs that will receive resources are those with the best channel conditions.

\subsubsection{Served users}

Figure 11 shows the served users by the DPSA-Tabu scheduler. It is clearly observed that this proposed scheduler serves an interesting number of UEs among the total number: in each TTI, the DPSA-Tabu serves most of GBR packets and gives them all their requirements.

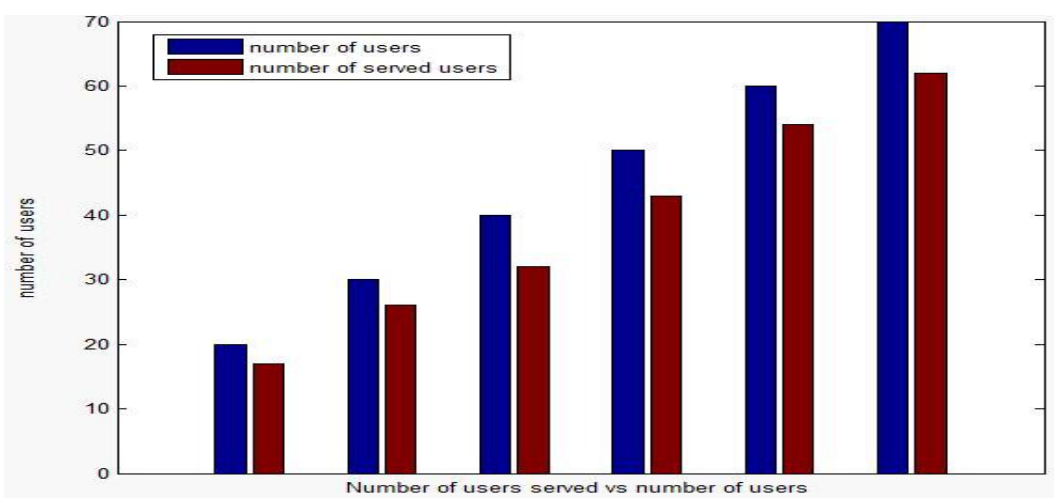

Fig.11. Served users by DPSA-Tabu 


\section{Conclusion}

In this paper, we proposed a novel scheduling algorithm named DPSA-Tabu. The DPSA-Tabu aims to maximize the system's sum throughput, to allow a fair distribution of available RBs and to handle GBR and NGBR traffic in LTE downlink systems. The performance of this algorithm is evaluated, considering LTE configuration parameters. The System performance was evaluated using simulations. The Simulation results prove that the DPSA-Tabu allows a better distribution of available RBs between the UEs while at the same time keeping the system's capacity utilization as good as possible. As a future work, we propose to further focus on evaluating QoS parameters for multimedia traffic and handle this algorithm in an LTE-A environment.

\section{References}

1. LTE; Evolved Universal Terrestrial Radio Access Access (E-UTRA); LTE physical layer; General description (3GPP TS 36.201 version 11.1.0 Release 11). February 2013.

2. 3GPP. E-UTRA and E-UTRAN overall description, Stage 2, TS 36.300 V8.10.0, 2009Y.

3. 3GPP. Evolved Universal Terrestrial Radio Access (E-UTRA) : Physical Channels and Modulation TS 136211 V11.5.0 (2014-01).

4. LTE; Evolved Universal Terrestrial Radio Access (E-UTRA) and Evolved Universal Terrestrial Radio Access Network (E-UTRAN); Overall description; Stage 2 3GPP TS 36.300 version 11.7.0 Release 11), (2013-09).

5. H.A.M. Ramli, R. Basukala, K. Sandrasegaran, and R. Patachaianand. Performance of well known packet scheduling algorithms in the downlink 3GPP LTE system. In IEEE 9th Malaysia International Conference on Communications (MICC), Dec. 2009. pp. 815 - 820.

6. M. Sajid Mushtaq, B.Augustin and A.Mellouk . QoE-Based LTE Downlink Scheduler for VoIP, Wirless communications and Networking Conference (WCNC), IEEE (2014). pp. $2190-2195$

7. S. Lee, S. Choudhury, A. Khoshnevis, S. Xu, S. Lu. Downlink MIMO with FrequencyDomain Packet Scheduling for 3GPP LTE INFOCOM 2009, IEEE (2009). pp. 1269 1277.

8. M. Sajid Mushtaq,S. Fowler, A.Mellouk and B.Augustin. QoE/QoS-aware LTE downlink scheduler for VoIP with power saving, Journal of Network and Computer Applications, 2014.

9. Lin Yan, Yue Guangxin.Channel-adapted and buffer-aware packet scheduling in LTE wireless communication system. In: 4th international conference on wireless communications, networking and mobile computing (WiCOM), October 2008. pp. 1-4.

10. Delgado O, Jaumard And B. Joint Admission Control and Resource Allocation with GoS and QoS in LTE uplink. IEEE GLOBECOM workshops. December 2010. pp.829-933.

11. Jha SC, Koç AT, Vannithamby R. Optimization of Optimization of mobile internet applications over LTE. In: IEEE Vehicular Technology Conference (VTC Fall) ). September 2012.

12. LTE; Evolved Universal Terrestrial Radio Access (E-UTRA); Medium Access Control (MAC) protocol specification (3GPP TS 36.321 version 9.3.0 Release 9), ETSI TS136 321, 2010. 
13. Capozzi, F. Piro, G. Grieco, L.A. Boggia, G. Camarda, P. Downlink Packet Scheduling in LTE Cellular Networks: Key Design Issues and a Survey. Communications Surveys \& Tutorials, IEEE (Volume:15, Issue: 2 ). 2013. pp. 678-700.

14. R. Khdhir, K. Mnif , L. Kamoun and L. Nuaymi,” New scheduling algorithm for Uplink LTE System, International Symposium on Network Computing (ISNCC),2014

15. L. Suk-Bok, L Pefkianakis, A Meyersonand, Shugong Xu, and Lu Songwu. Proportional fair frequency-domain packet scheduling for 3gpp LTE. INFOCOM ,IEEE, pp. 2611-2615, 2009

16. M Laguna, J Barnes, and F Glover. Intelligent scheduling with tabu search: An application to jobs with linear delay penalties and sequence dependent setup costs and times. Journal of Applied Intelligence, 1993. pp. 159-172

17. M. Pilegaard Hansen. Tabu search for multio bjective optimization: Mots. MCDM 97, Cape Town, pp. 6-10, January, 1997.

18. Technical specification group radio access networks - Deployment aspects. 3GPP TR 25.943.

19. S. Haidar, W. El-Hajj, and K. Tohme. A QoS-Aware Uplink Scheduling Paradigm for LTE Network. International Conference on Advanced Information Networking and Applications, IEEE, pp. 1097- 1104, 2013.

20. R. Jain, D. M. Chiu, and W. Hawe, "A Quantitative Measure of Fairness and Discrimination for Resource Allocation in Shared Systems," Technical Report TR-301, DEC Research Report, 1984. 